\title{
Case Report on Long COVID-19
}

\author{
Ganesh Kamath ${ }^{1}$, Muthukumar Mani², Nagarathna Poojary',**
}

${ }^{1}$ Department of General medicine, Dr. N. R. Acharya Memorial Hospital, Udupi, Karnataka, INDIA.

2Department of Pharmacy Practice, Faculty of Pharmaceutical Science, PES University, Bangalore (formerly as PES College of Pharmacy), Karnataka, INDIA.

\begin{abstract}
COVID-19 has dominated the whole world and resulted in increased morbidity and mortality. Here we present a case report of 54-years-old man with repeated admissions to the hospital after severe COVID-19 pneumonia which on progression lead to sepsis with bronchitis. There was Dexamethasone induced hyperglycaemia and was administered with antibiotics, corticosteroids, anti-asthmatics and COPD preparations. He was recurrently admitted to hospital for 4 times and was on oxygen support. These recurrent admissions lead to decreased quality of life and increased healthcare cost. Polypharmacy was on peak and underlying condition was not subsided. Prompt diagnosis and early interventions regarding COVID-19 complications must include a good scientific approach.
\end{abstract}

Key words: Pneumonia, Bronchitis, COVID-19, Sepsis, Hyperglycemia, Polypharmacy.

\section{INTRODUCTION}

Coronavirus disease 2019 (COVID-19) emerged in Wuhan city in December 2019 caused by the severe acute respiratory syndrome coronavirus 2 (SARS-CoV-2) rapidly spread throughout China resulted in varying degrees of illness. ${ }^{1,2}$ SARS-CoV-2 has dominated the whole world population by fast transmission and high mortality rate. ${ }^{3,4}$ Infected individuals present with life-threatening diseases characterized by acute respiratory distress syndrome (ARDS), sepsis, multisystem organ failure, cytokine storm, thromboembolic disease and extra pulmonary manifestations. ${ }^{5,6}$

Intensive spread of COVID-19 has resulted in high rates of ignored diagnosis of viral sepsis. Hence it is required to evaluate the clinical characteristics and risk factors associated with viral sepsis. ${ }^{7}$ During infection, a heterogenous syndrome due to a dysregulated host response reflect as a sepsis which is associated with organ dysfunction. ${ }^{14,8}$ Pathophysiology, clinical manifestations as well as autopsy examinations of the host inflammatory response should be reviewed as a consequence of sepsis induced by virus and/or other pathogens. Pathogenesis involves dysregulated inflammation, vascular dysfunction, pulmonary vascular endothelialitis, angiogenesis, thrombosis and respiratory complications. It is understood that coagulopathy, cytokine storm, hyper inflammation are the contributors of disease severity. Along with supportive therapy, immunomodulatory therapies should also be considered. 5, $, 10,3,11,4,12,8$ Endothelial injury leads to release of certain molecules which contributes to the diagnosis as well as prognostic biomarkers of infection. ${ }^{13}$ A sepsis intervention protocol (SIP) can be useful in septic shock through the use of a sepsis checklist, compliance with bundle elements like "resuscitation" and "management". ${ }^{14}$

\section{CASE PRESENTATION}

A 54-years-old male presented with fever, body ache, abdominal pain, cough with expectoration, nausea, chest pain and general
DOI: 10.5530/ijopp.14.4.67

Address for correspondence: Ms. Nagarathna Poojary, Department of Pharmacy Practice, PES University, (formerly PES College of Pharmacy), Bangalore, Karnataka, INDIA.

Phone no: +919148324479 Email id: nagipoojarypgs@ gmail.com

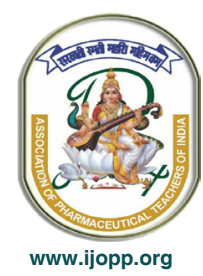


Table 1: Laboratory Values of the patient in hospital admissions. The first column represents the lab parameters and the second and third columns list the lab values on the first and third admission in hospital. Last column represents reference values.

\begin{tabular}{|c|c|c|c|}
\hline Parameter & $\begin{array}{c}1^{\text {st }} \text { admission } \\
10 / 04 / 2021\end{array}$ & $\begin{array}{c}3^{\text {rd }} \text { admission } \\
1 / 06 / 2021\end{array}$ & Reference \\
\hline \multicolumn{4}{|l|}{ Hematology } \\
\hline Hemoglobin(g/dl) & 14.5 & 10.9 & $12-14$ \\
\hline Total count(cu/mm) & 8500 & 11900 & $4000-11000$ \\
\hline Neutrophil (\%) & 82 & 70 & $02-07$ \\
\hline Lymphocytes (\%) & 16 & 28 & $20-40$ \\
\hline Platelet count(lakh/cu/mm) & 1.8 & 3.3 & $1.5-4.0$ \\
\hline PCV (\%) & 43.42 & 32.52 & $30-50$ \\
\hline RBC count(lakh/cu/mm) & 5.68 & 4.12 & $4.2-5.4$ \\
\hline MCV (\%) & 77 & 79.0 & $76-96$ \\
\hline $\mathrm{MCH}(\%)$ & 25.6 & 26.5 & $27-32$ \\
\hline $\mathrm{MCHC}(\%)$ & 33.4 & 33.5 & $32-36$ \\
\hline \multicolumn{4}{|l|}{ Biochemistry } \\
\hline RBS (mg/dl) & 120 & 225 & $60-180$ \\
\hline Serum CRP (mg/dl) & 12.9 & 101.5 & $0.0-6.0$ \\
\hline \multicolumn{4}{|l|}{ Blood urea and serum creatinine } \\
\hline Blood urea(mg/dl) & 30 & 26 & $10-40$ \\
\hline Serum creatinine(mg/dl) & 1.1 & 0.8 & $0.4-1.5$ \\
\hline MP KIT & Negative & Negative & Negative \\
\hline Dengue & Negative & Negative & Negative \\
\hline Aerobic culture test & Negative & Negative & Negative \\
\hline
\end{tabular}

PCV-Packed Cell Volume; RBC-Red Blood Cells; MCV-Mean Corpuscular Volume; MCH-Mean Corpuscular Haemoglobin; MCHC-Mean Corpuscular Haemoglobin Concentration; RBS-Random Blood Sugar; CRP-C Reactive Protein; MP KIT-Malaria Antigen Test Kit

weakness for 3-4 days. He had a past medical history of hyperlipidaemia and renal calculi which subsided after the therapy. Reverse Time Polymerase Chain Reaction (RT-PCR) of nasopharyngeal swabs using the Quant Inova Probe RT-PCR kit was performed..$^{15}$ Complete blood count, MP Kit (Malarial Parasite), RBS (Random Blood Sugar), Dengue, Blood urea, Serum creatinine tests were done (Table 1). There were increased levels of granulocytes and C-reactive protein.

Immediately his chest X-RAY was taken, which showed sepsis with bronchitis (Figure 1). Differential diagnosis of viral pneumonia was done. After 2 days, the results of RT-PCR came positive. Spiral CT sections of chest were obtained with sagittal and coronal reconstruction which showed bilateral multifocal peripheral, sub pleural and non-lobar distribution of ground glass opacities in the lobes of both lung fields (Table 2). Approximately $>10 \%$ of lung parenchyma was involved with CT severity score of $9 / 25$ (moderate disease). With all these evidence, patient's condition was diagnosed as Atypical Viral Pneumonia-COVID-19 infection (CO-RADS 6). The

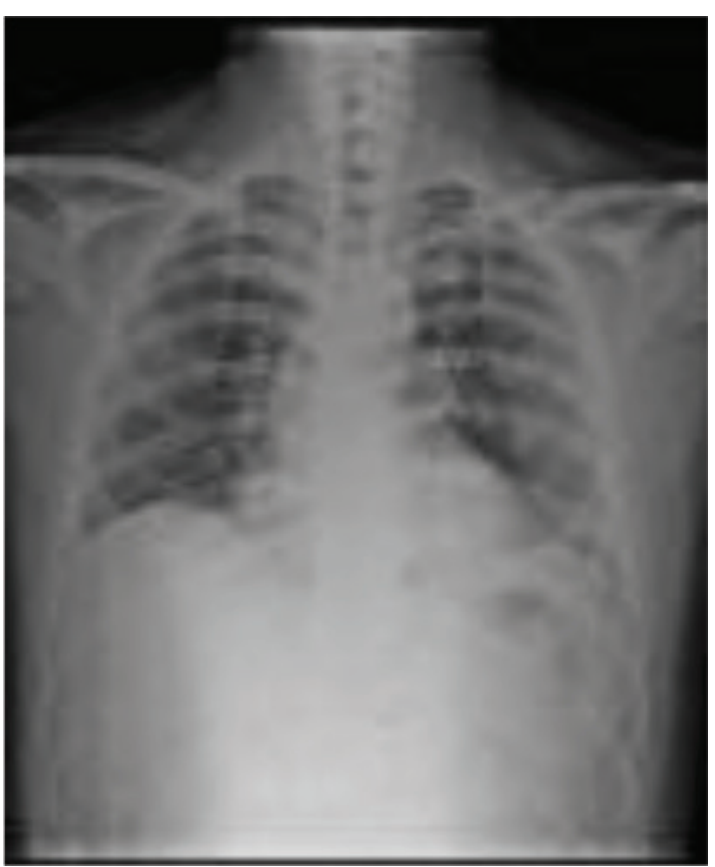

Figure 1: 54-year's-old man with COVID-19 who presented with chest pain and cough with expectoration on 10/04/2021 a) Pneumoperitoneum b) Bilateral extensive fibrosis. 


\section{Table 2: CT Severity Score (CTSS).}

\begin{tabular}{ccc} 
Lung lobes & $\begin{array}{c}\text { Percentage } \\
\mathbf{1 3 / 0 4 / 2 0 2 1}\end{array}$ & $\begin{array}{c}\text { Percentage } \\
\mathbf{1 8 / 0 5 / 2 0 2 1}\end{array}$ \\
\hline Right upper lobe & $>15 \%$ & $20 \%$ \\
Right mid lobe & $<5 \%$ & $30 \%$ \\
Right lower lobe & $<10 \%$ & $80 \%$ \\
Left upper lobe & $<5 \%$ & $40 \%$ \\
Left lower lobe & $<5 \%$ & $80 \%$ \\
CTSS (overall) & 9 out of 25 (moderate) & 17 out of 25 \\
\hline
\end{tabular}

patient was treated with IV Fluid-NS 3 points, Piperacillin sodium+Tazobactam $(4 \mathrm{~g}+0.5 \mathrm{~g}) \mathrm{IV}$, Pantoprazole $40 \mathrm{mg}$ slow IV, Ondansetron 1amp IV, Diclofenac 1amp deep IM SOS, T.Supradyn (vitamin supplements), Deriphylline 1amp IV. On second day T.Favipiravir 400mg, Dexamethasone 2cc IV, T.Ivermectin $12 \mathrm{mg}$, T.Dabigatran Etexilate $110 \mathrm{mg}$, Oxygen inhalation 2 litres/minute were added to the prescription. On $3^{\text {rd }}$ day he had right side chest pain. Oxygen was made SOS and the frequency of Favipiravir was made to 4-0-4. On $4^{\text {th }}$ day, he complained of chest pain and a 12 lead ECG was taken. Ondansetron was made twice daily. On $5^{\text {th }}$ day he had cough with breathlessness. T.Dabigatran Etexilate $110 \mathrm{mg}$ was stopped and Enoxaparin $0.4 \mathrm{mg}$ IV was added to the prescription. On $6^{\text {th }}$ day he again complained of cough with severe breathlessness. T. Acebrophylline+acetylcysteine $(100 \mathrm{mg}+600 \mathrm{mg})$ was added to the prescription and ondansetron was stopped. On the $7^{\text {th }}$ day he had severe hypoxia and was in need of a ventilator. He was referred to another hospital for ventilator support. His RBS was increasing and was diagnosed to have Dexamethasone induced hyperglycaemia. Later he was treated for 22 days and discharged with T.Prednisolone, T.Dabigatran Etexilate, T.Cephalexin, T.Telmisartan and T.Metformin+Glipizide.

Within 8 days of discharge, the patient was readmitted to the hospital with complaints of breathlessness, generalized weakness and nausea. Chest X-Ray was taken which reflected bilateral peripheral infiltration (Figure 2). HRCT Thorax (High Resolution Computed Tomography) revealed patchy airspace consolidation with ground glass haziness in bilateral lungs viz.apical anterior and posterior segments of right upper lobe, lateral and medial segments of right middle lobe, apical and basal segments of right lower lobe, apical posterior, anterior and lingular segments of left upper lobe, apical and basal segments of left lower lobe (Table 2). RTPCR was negative, and the findings were concerning for Typical SARS-CoV2 Pneumonia. Abdominal CT scan showed evidence of Extensive Pneumoperitoneum secondary to high flow oxygen, Bilateral renal calculi and Grade I Prostatomegaly. Finally he was diagnosed as Post COVID-19 Pneumothorax Hypoxia and in this admission the patient was treated with Cefoperazone+Sulbactam $(1 \mathrm{~g}+0.5 \mathrm{~g}) \mathrm{IV}$, Pantoprazole $40 \mathrm{mg}$ slow IV, Ondansetron $1 \mathrm{amp}$ IV, Deriphylline 1amp IV, Dexamethasone 2cc IV, T.Acebrophylline+acetylcysteine $(100 \mathrm{mg}+600 \mathrm{mg})$, Diclofenac lamp deep IM SOS, Oxygen inhalation 2 litres/minute. On $2^{\text {nd }}$ day Capsule Beminal Forte was added. On $3^{\text {rd }}$ day he had complaints of constipation and his random blood sugar value was higher. T.Dulcolex was given to ease defecation. T.Glimepiride $4 \mathrm{mg}$, Cremaffin Plus Syrup, T.Pirfenidone $200 \mathrm{mg}$ were added to the prescription. On $6^{\text {th }}$ day, he had cough with weakness. Ondansetron and Dexamethasone was stopped. On $7^{\text {th }}$ day T.Glimepiride $4 \mathrm{mg}$ was stopped and he had hypoglycemia. Proton-SF protein powder 2teaspoon was prescribed and discharged after 8 days of treatment.

After 14 days the patient was again admitted to hospital with complaints of fever, body ache, giddiness, cough with expectoration and weakness for 2 days. Haematological tests revealed high blood sugar and culture report showed negative results for microbial infections (Table 1). RT PCR was also negative for COVID-19. He had fever almost all the days of his hospital stay and had loss of appetite on $13^{\text {th }}$ and $14^{\text {th }}$ day. IV Fluid-NS 2 points, Piperacillin sodium+Tazobactam $(4 \mathrm{~g}+0.5 \mathrm{~g})$ IV, Pantoprazole 40mg slow IV, Ondansetron lamp IV, Deriphylline 1amp IV, Oxygen inhalation 2 litres/

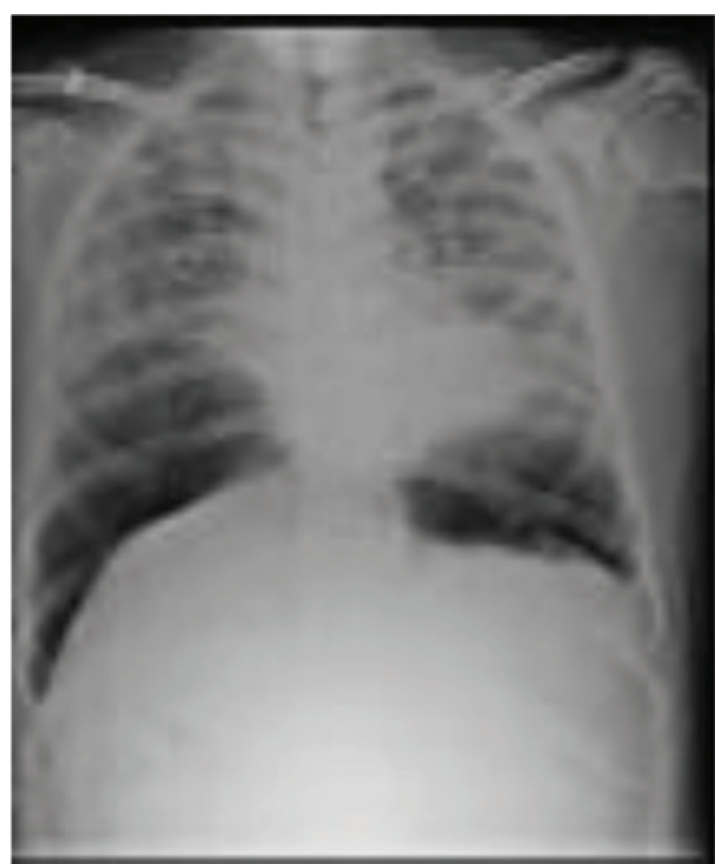

Figure 2: 54-year's-old man with past medical history of COVID-19 who presented with breathlessness on 16/05/2021 a) bilateral peripheral infiltration suggestive of viral pneumonia. 
minute, T.Pirfenidone $200 \mathrm{mg}$, Proton-SF protein powder 2 teaspoon, Diclofenac 1amp deep IM SOS, T.GMPS, T.Betahistine $16 \mathrm{mg}$, T.Meropenem $300 \mathrm{mg}$, T.Cinnarizine $25 \mathrm{mg}$ were administered and was discharged after 15 days of hospital stay.

\section{DISCUSSION}

SARS CoV-19 infected patients are recovered mostly within a few weeks of time even if the patient with severe infection also recovers completely. Few COVID-19 infected patients suffer with symptoms for a long time after initial recovery, regardless of severity of infection. ${ }^{16}$ In our case the patient got readmitted to the hospital for 2 times for the management of post Covid symptoms like breathlessness, cough and fever (Figure 3). There were also elevated levels of neutrophils which are the primary central contributors in immune responses to resist pathogen invasion. ${ }^{17}$ NICE guidelines define patients with symptomatic COVID-19 from 4 to 12 weeks as Long COVID-19 cases. In the above case the patient also has persistent symptoms of COVID-19 for more than 24 days after recovery from acute COVID-19. ${ }^{18}$

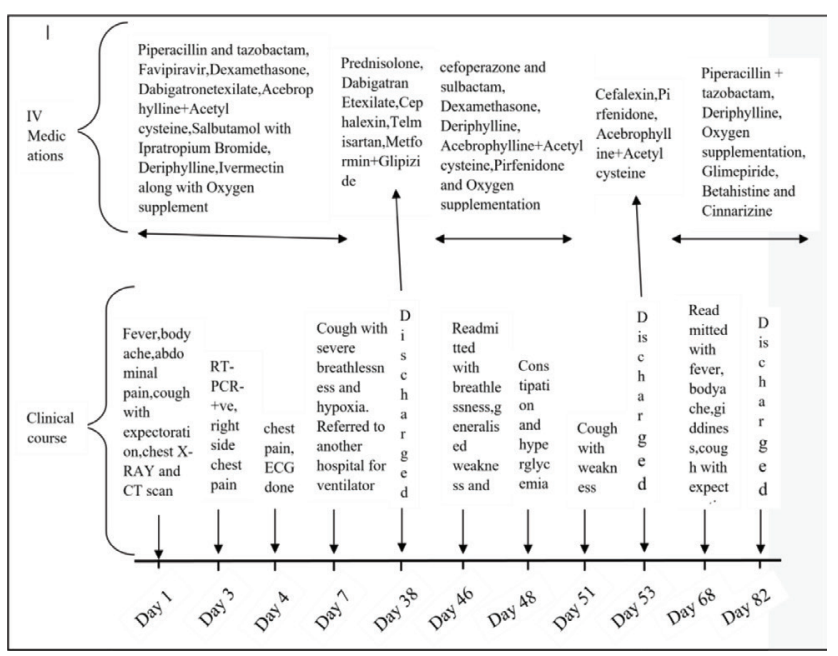

Figure 3: Clinical course of the patient.

Patient perspective: Initially I was distressed about repeated admissions to the hospital. Out of COVID-19 fear, I suffered both physically and mentally. Even after the COVID-19 recovery period, I was getting additional symptoms which made me difficult to perform my daily activities. My family members were also tensed about my repeated admissions which increased my financial burden also. I think the challenges which I faced must be reported so that early diagnosis and treatment of reinfection co-infections reduce prolonged sufferings from COVID-19.

Informed consent: Patient was informed about the reporting of his medical condition and the consent is attached.
Our case report also reveals that both cell-mediated and humoral responses activated immunity is uncertain for the preventive role for COVID-19 reinfection. High flow oxygen favours pneumoperitoneum and secondary bacterial infections. It also gives rise to the hypothesis that the immunomodulatory effect of corticosteroid explains the possible mechanism for reinfection of COVID-19 which is responsible for the second admission of the patient and also the patient was presented with Dexamethasone induced hyperglycaemia. During the third admission patient has persistent symptoms like cough, fever and laboratory data also revealed increased inflammatory action. But the Blood culture report was negative for aerobic bacteria. However, there is no confirmatory evidence to support sepsis.

\section{CONCLUSION}

In conclusion, the case reveals that COVID-19 infection can cause reinfection and also collapse the inflammatory cascade of the patients. The case also explains the importance of healthcare interventions and continuous monitoring of health of the COVID-19 infected patients even after acute COVID-19 recovery. There should be a systematic approach to suspect any complication that persists after COVID-19. Repeated admissions worsen the patient's health as well as the financial condition.

\section{ACKNOWLEDGEMENT}

We are thankful to Dr.N.R.Acharya Memorial Hospital Udupi, Prof Dr. Srinivasan R (Chairperson, Department of Pharmacy Practice, PES University, Bangalore) and Prof Dr. Saravanan J (Dean, faculty of pharmaceutical sciences, PES University, Bangalore.) for their support and guidance.

\section{CONFLICT OF INTEREST}

The authors declare that there is no conflict of interest.

\section{ABBREVIATIONS}

COPD: Chronic Obstructive Pulmonary Disorder; SARS CoV-2: Severe Acute Respiratory Syndrome Coronavirus2; RT PCR: Reverse Transcriptase Polymerase Chain Reaction; MP Kit: Malarial Parasite; CO-RADS 6: COVID-19 Reporting and Data System category 6; HRCT: High Resolution Computed Tomography. 


\section{SUMMARY}

A 54-years male patient came to hospital with complaints of fever, body ache, abdominal pain, nausea, chest pain, general weakness, and cough with expectoration. $\mathrm{He}$ was administered with Piperacillin with Tazobactam antibiotics, Favipiravir, Dexamethasone, Acebrophylline +Acetylcysteine, Salbutamol with Ipratropium Bromide, Deriphylline, Ivermectin along with Oxygen supplement. Due to the requirement of ventilator, he was shifted to another hospital and there he got discharged with Prednisolone, Dabigatran Etexilate, Cephalexin, Telmisartan, Metformin+Glipizide. Within 8 days of discharge, the patient was readmitted to the hospital with complaints of breathlessness, generalized weakness and nausea. HRCT Thorax (High Resolution Computed Tomography) revealed patchy airspace consolidation and concluded with typical SARS CoV-2 pneumonia. Finally, he was diagnosed as Post COVID-19 Pneumothorax Hypoxia and in this admission the patient was treated with cefoperazone and sulbactam, Dexamethasone, Deriphylline, Acebrophylline +Acetylcysteine, Pirfenidone and Oxygen supplementation. After 14 days the patient was again admitted to hospital with complaints of fever, body ache, giddiness, cough with expectoration and weakness for 2 days. Blood culture tests reported no organisms. He was treated with Piperacillin + tazobactam, Deriphylline, Oxygen supplementation.

\section{REFERENCES}

1. Phillipson $M$, Kubes $P$. The neutrophil in vascular inflammation. Nat Med. 2011;17(11):1381-90. doi: 10.1038/nm.2514, PMID 22064428.

2. Mehta P, McAuley DF, Brown M, Sanchez E, Tattersall RS, Manson JJ, HLH Across Speciality Collaboration, UK. COVID-19: consider cytokine storm syndromes and immunosuppression. Lancet. 2020;395(10229):1033-4. doi: 10.1016/S0140-6736(20)30628-0, PMID 32192578.

3. Zhou F, Yu T, Du R, Fan G, Liu Y, Liu Z, et al. Clinical course and risk factors for mortality of adult inpatients with COVID-19 in Wuhan, China: a retrospective cohort study. Lancet. 2020;395(10229):1054-62. doi: 10.1016/S01406736(20)30566-3, PMID 32171076.

4. Chan JF, Yip CC, To KK, Tang TH, Wong SC, Leung KH, et al. Improved Molecular Diagnosis of COVID-19 by the Novel, Highly Sensitive and Specific
COVID-19-RdRp/Hel Real-Time Reverse Transcription-PCR Assay Validated in vitro and with Clinical Specimens. J Clin Microbiol. 2020;58(5):e00310-20. doi: 10.1128/JCM.00310-20, PMID 32132196.

5. Grasselli G, Zangrillo A, Zanella A, Antonelli M, Cabrini L, Castelli A, et al. COVID-19 Lombardy ICU Network. Baseline characteristics and outcomes of 1591 patients infected with SARS-CoV-2 admitted to ICUs of the Lombardy region, Italy. JAMA. 2020;323(16):1574-81. doi: 10.1001/jama.2020.5394, PMID 32250385.

6. Moore JB, June CH. Cytokine release syndrome in severe COVID-19. Science. 2020;368(6490):473-4. doi: 10.1126/science.abb8925, PMID 32303591.

7. Fu L, Wang B, Yuan T, Chen X, Ao Y, Fitzpatrick T, et al. Clinical characteristics of coronavirus disease 2019 (COVID-19) in China: A systematic review and meta-analysis. J Infect. 2020;80(6):656-65. doi: 10.1016/j.jinf.2020.03.041, PMID 32283155.

8. Ackermann M, Verleden SE, Kuehnel M, Haverich A, Welte T, Laenger F, et al. Pulmonary vascular endothelialitis, thrombosis, and angiogenesis in Covid-19. N Engl J Med. 2020;383(2):120-8. doi: 10.1056/NEJMoa2015432, PMID 32437596.

9. Faqihi F, Alharthy A, Alodat M, Kutsogiannis DJ, Brindley PG, Karakitsos D. Therapeutic plasma exchange in adult critically ill patients with life-threatening SARS-CoV-2 disease: A pilot study. J Crit Care. 2020 Dec;60:328-333. doi: 10.1016/j.jcrc.2020.07.001. Epub 2020. PMID: 32763058.

10. Igiebor O, Nakeshbandi M, Mehta N, Ozaki R, Lucchesi M, Daley M, et al. Impact of sepsis intervention protocol (SIP) on adherence to three-hour and six-hour bundles and mortality outcomes in the emergency department. Int J Clin Res Trials. 2020;5(2):149. doi: 10.15344/2456-8007/2020/149, PMID 32832739.

11. Lin HY. The severe COVID-19: A sepsis induced by viral infection? and its immunomodulatory therapy. Chin J Traumatol. 2020;23(4):190-5. doi: 10.1016/j. cjtee.2020.06.002, PMID 32690231.

12. Beltrán-García J, Osca-Verdegal R, Pallardó FV, Ferreres J, Rodríguez M, Mulet S, et al. Sepsis and coronavirus Disease 2019: common features and anti-inflammatory therapeutic approaches. Crit Care Med. 2020;48(12):1841-4. doi: 10.1097/CCM.0000000000004625, PMID 32826431.

13. Zafer MM, El-Mahallawy HA, Ashour HM. Severe COVID-19 and sepsis: immune pathogenesis and laboratory markers. Microorganisms. 2021;9(1):159. doi: 10.3390/microorganisms9010159, PMID 33445583.

14. Venkatesan P. NICE guideline on long COVID. Lancet Respir Med. 2021;9(2):129. doi: 10.1016/S2213-2600(21)00031-X, PMID 33453162.

15. Tang G, Luo Y, Lu F, Li W, Liu X, Nan Y, et al. Prediction of sepsis in COVID-19 using laboratory indicators. Front Cell Infect Microbiol. 2020;10:586054. doi: 10.3389/fcimb.2020.586054.

16. Chen B, Han J, Chen S, Xie R, Yang J, Zhou T et al. Micro. Let-7b regulates neutrophil function and dampens neutrophilic inflammation by suppressing the canonical TLR4/NF-kB pathway. Front Immunol. 2021;12. PMID 653344.

17. Mayo clinic. COVID-19 (coronavirus): long-term effects [cited 3-7-2021]. Available from: https://www.mayoclinic.org/diseases-conditions/coronavirus/indepth/coronaviruslong-term-effects/art-20490351.

18. Kaur S, Hussain S, Kolhe K, Kumar G, Tripathi DM, Tomar A, et al. Elevated plasma ICAM1 levels predict 28-day mortality in cirrhotic patients with COVID-19 or bacterial sepsis. JHEP Rep. 2021;3(4):100303. doi: 10.1016/j. jhepr.2021.100303. PMID 33997748. 